\title{
Geleitwort \\ zu dem unveränderten Nachdruck 1957
}

Das gegenwärtige Erscheinen des 2. Bandes des Warenkaufs hat die Frage nach der Behandlung des 1. Bandes entstehen lassen. Er ist seit langem vergriffen. Nun, da der 2. Band erworben werden kann, wird der Wunsch, auch den 1. Band und damit das vollständige Werk zu besitzen, vielfach besonders dringlich werden. Ist das ein ausreichender Anlaß, den 1. Band unverändert neu zu drucken? Dagegen könnte die Erwägung sprechen, daß die Darstellung inzwischen in manchen Punkten übcrholt ist. Seit dem Jahre 1936 sind das griechische ZGB v. 1940, der neue italienische Codice Civile v. 1942 und Kodifikationen jüngeren Datums in einigen latein-amerikanischen Ländern in Kraft getreten. In England wurde der für Leistungshindernisse bedeutsame Frustrated Contracts Act, 1942 (6\& 7 Geo. 6, c. 4.) erlassen. Lnd in Österreich gilt nicht mehr das aHGB, sondern das deutsche Handelsgesetzbuch. Rechtsprechung und Rechtslehre haben sich weiter entwickelt und neue Gesichtspunkte gewonnen. Trotzdem scheint ein unveränderter Neudruck die beste Lösung zu sein. Denn eine Durchsicht des 1 . Bandes zeigt, da $B$ die von Rabel entfalteten Gedankengänge durch die mehrfachen Änderungen der positiv-rechtlichen Daten ihre Bedeutung und Aktualität nicht eingebüßt haben. So würde der bei einer Korrektur des 1. Bandes entstehende erhebliche Zeitverlust schwerlich zu rechtfertigen sein. Der 1. Band wird also in einem unveränderten Neudruck hiermit vorgelegt. Da der 2. Band im Anhang den Bericht Rabels über die Haager Konferenz zur Vereinheitlichung des Kaufrechts von 1951 enthält, dürfte auch eine Art Brückenschlag zwischen dem älteren und dem neueren Teil des Gesamtwerkes durch den Autor selbst geschehen sein.

Uber die neuere Literatur kann sich der Leser im 2. Band unterrichten.

Herrn Otto A. Friedrich, dem Vorsitzenden des Vorstands der Phoenix-Gummi-Werke AG., Hamburg-Harburg, danke ich auch an dieser Stelle aufrichtig für die Finanzierung des vorliegenden Neudrucks.

Hamburg, im Mai 1957

\section{Hans Dölle}


